تم انجاز هذا البحث لغرض نحلية المياه الجوفية في جامعة نكريت باستخدام منظومة غشاء التناضح العكسي. استخدم غثاء

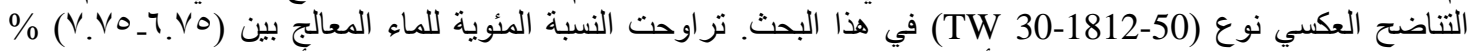

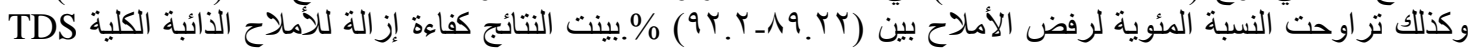

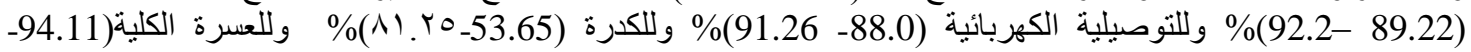

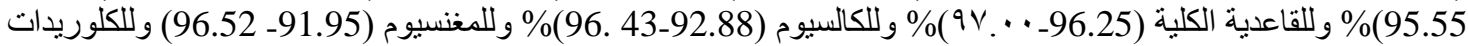

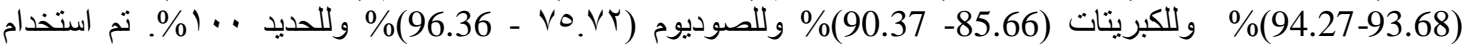
برنامج ROSA72 لتصميم منظومة تناضح عكسي للماء الجوفي الخام وكانت النتائج مرضية.

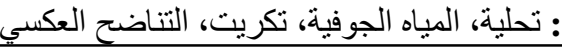

و المبادلات الأيونية (Ion exchange) و الفصل

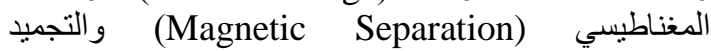
Chemical ) والترسيب (Freezing) (Precipitation Osmosis

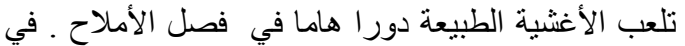

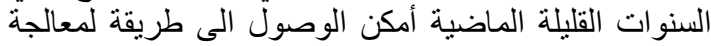
المياه المالحة باستخدام نظرية التناصح العكسي العيل (R.O)

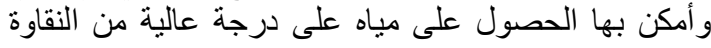

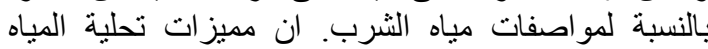

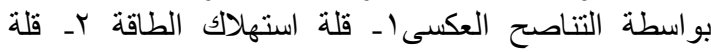

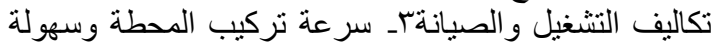

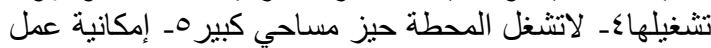
توسعات للححطة في أبي وقت جـ الـ تستخدم في تحلية المياه

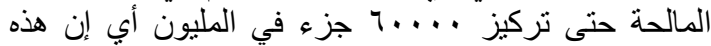
الطريقة تستخدم في تحلية مياه الآبار المالحة (Salt Water)

ومياه البحار ( Sea Water). التناضح أو التنافذ (باللغة الإنجليزية: Ossmosis)، هي هي

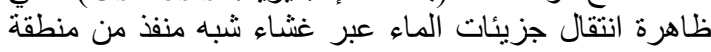

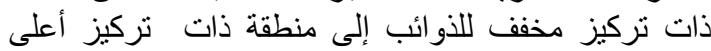

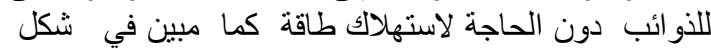
(1). الغثاء الثبه منفذة semi-permeable يسمح بناء بنفوذ الماء (الدذيب) ولا يسمح بنفوذ المذاب (solute) حتى الثى

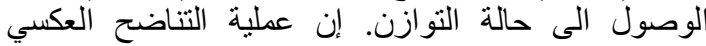

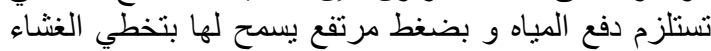

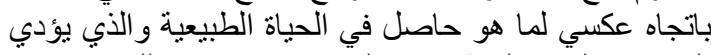

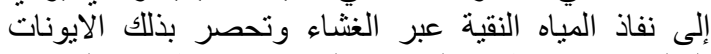
المالحة في منطقة ملوحة عالية .تعتمد طريقة التناضح

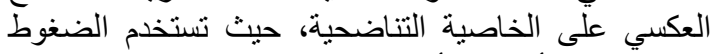
السلطة على أسطح الأغشية للتغلب على الضغط التناضحي
لقد أدى استنز اف الموارد الطبيعية مثل الوقود الاحفوري

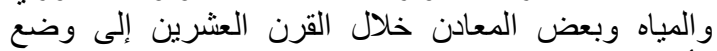

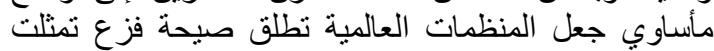

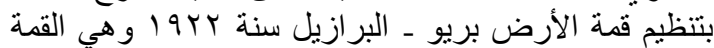

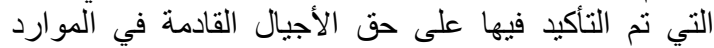

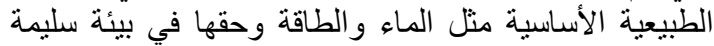

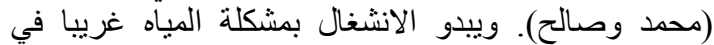

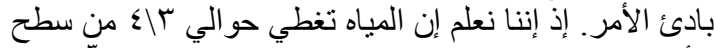

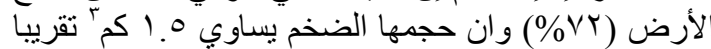

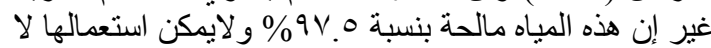

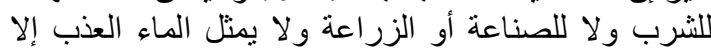

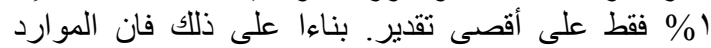

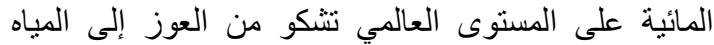
العذبة وان اللجوء إلى تحلية المياه المالحة (المياه الجوفية أو الويان

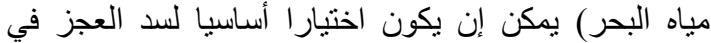

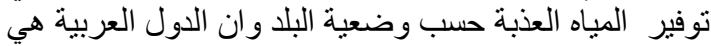

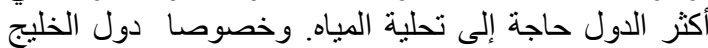

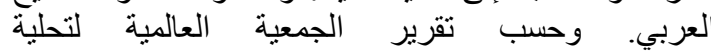
International Desalination Association- المياه

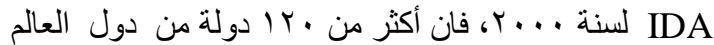

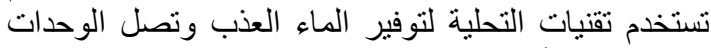

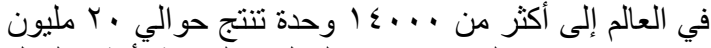
متر مكعب في اليوم وتعتبر الدول العربة العبية أكثر الدول

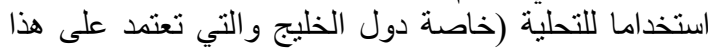

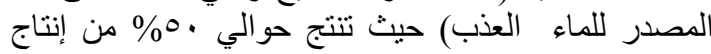

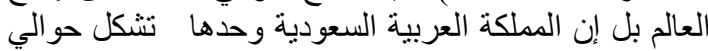
وبr من إنتاج العالم من ماء التحلية (محمد وصالح).

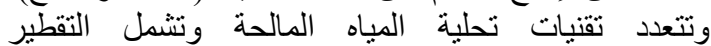
(Distillation )

* Corresponding author:

Dr. Abbas Hadi Abbas

$凶$ abbas.hadi@uosamarra.edu.iq 


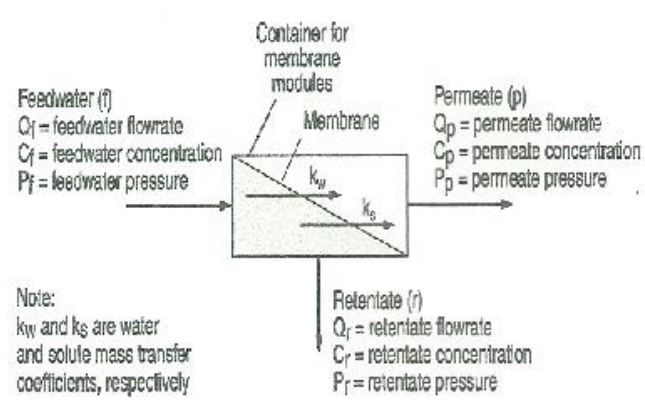

(2007 ( ): مخطط لغشاء التتاضح العكسي Takashi Asano (et.al 2007)

لحساب الضغط التتاضحي تسنخدم عدة معادلات ومنها هذه

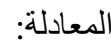
$\pi=\frac{0.0385 T D S(t+273)}{1000-\frac{T D S}{1000}}$

(1) (Ibrahim S. Al-Mutaz et.al)

حيث إن : $\pi$ الضغط التناضحي psi

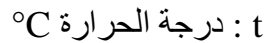
TDS : الأملاح الذائبة الكلية ملغمالتر : TDS

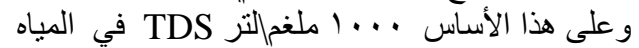
( المر اد تحليته يكافئ تقريبا $=$ =

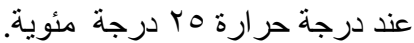

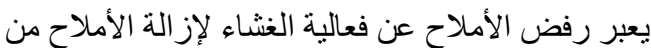
الماء ويمكن إن يحسب من العلاقة التالية.

Salt Rejection $\%=\left(1 \cdot \frac{\text { rroùust } 7 D S}{f_{\text {ged }} \text { TDS }}\right) \times 100 \ldots \ldots . . . .(2)$ (Takashi Asano et.al) إما معدل الاستعادة لماء التغذية لنظام التناضح العكسي يعطى بالمعادلة التالية

Rccovery ratc $\%=(q D) \times 100$

(3) (Tøkashi Asano et.al)

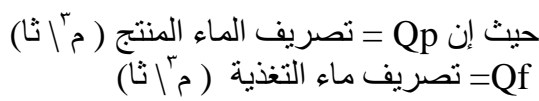

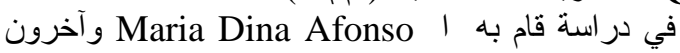

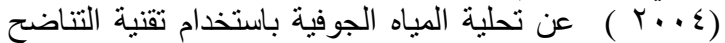

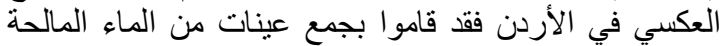

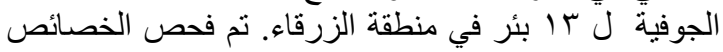

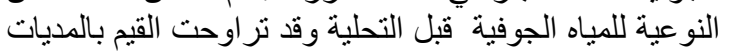

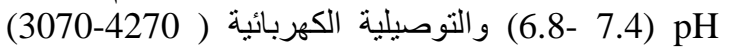
مايكرو موز اسم و TDS (62-0.4 (1632-3072) ملغمالتر

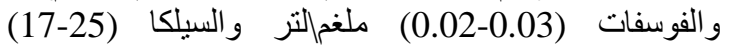

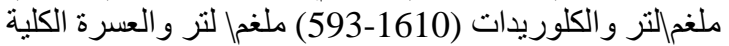

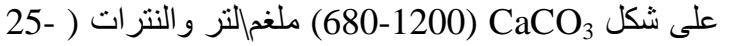

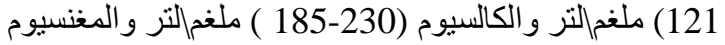

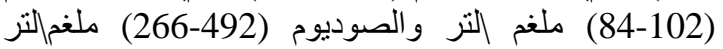

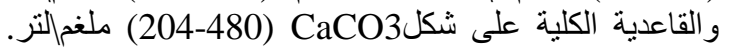

الطبيعي للماء، فإذا وضع غشاء شبه منفذ بين محلولين

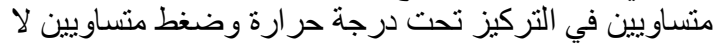

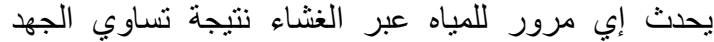

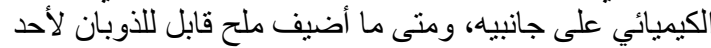

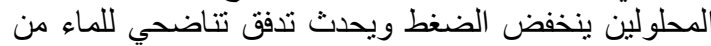

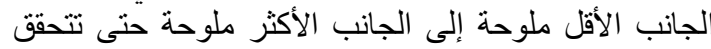

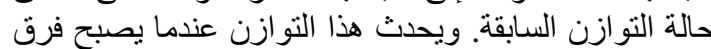

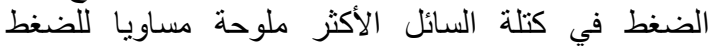

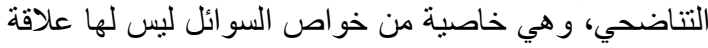

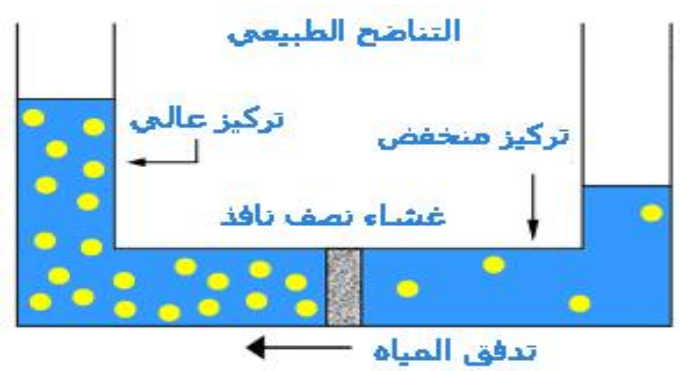

( ) ( ) (التناضح الطبيعي.

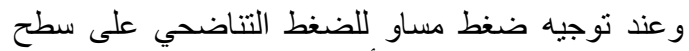

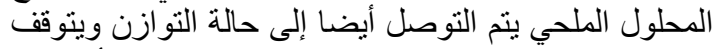

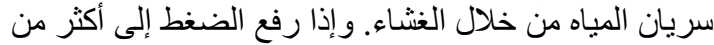

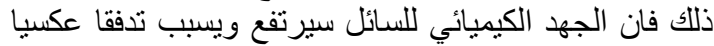

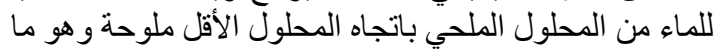

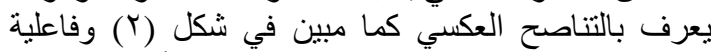

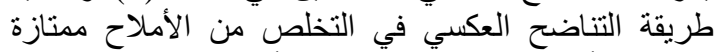
تصل إلى أكثر من 99\% وكنلك فان أغشية التناضح العكسي

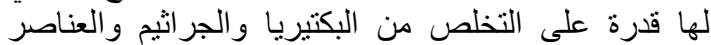

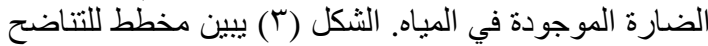

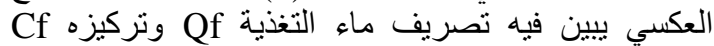

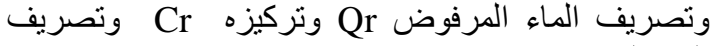

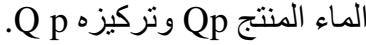

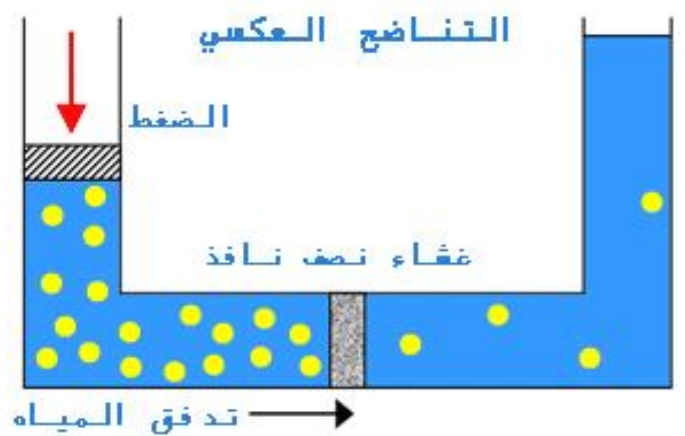

RO ( ) ( ) التناضح العكسي 
تم استخدام مرشح تناضح عكسي نوع LE-440 Film (BW30 Tec)

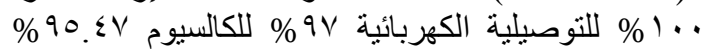

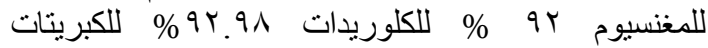

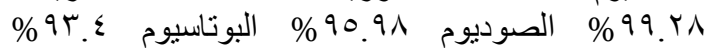

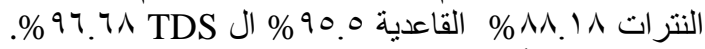

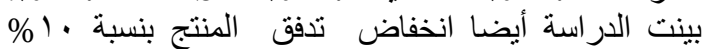

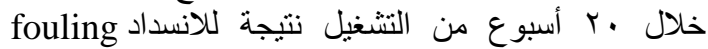
الحاصل بالغشاء.

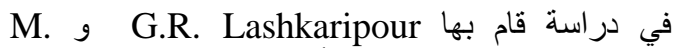

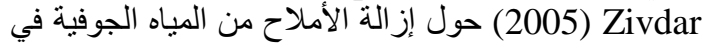
مدينة زاهدان في إيران باستخدام تقنتية التناضح العكسي.

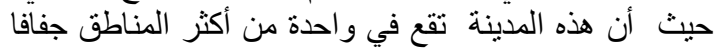

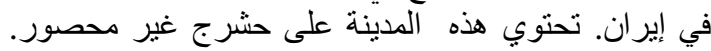

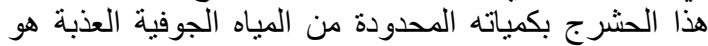

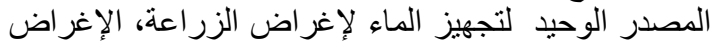

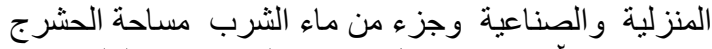

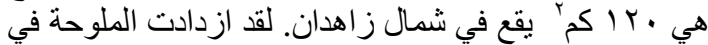
العقود الحالية نتيجة لقلة شحن المباه الجياه الجوفية و والتطور

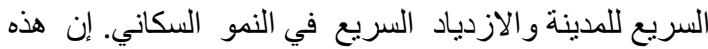

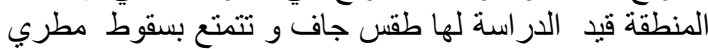

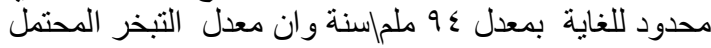

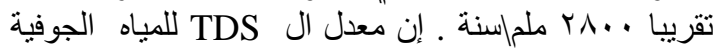

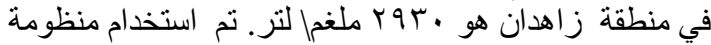

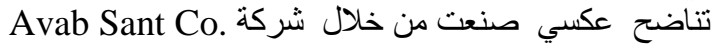

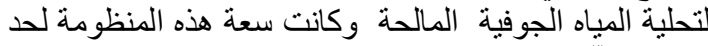
.

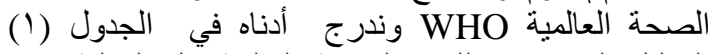
التحليل الكيمياوي للمياه الجوفية المالحة قبل التحلية وبعد

التحلية.
لقد نم استخدام غشاء نوع SW30-2521 ـ تم الحصول على الى

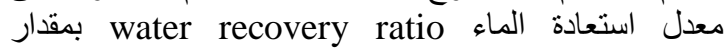
\% ونسبة رفض الأملاح RV.0 $\% 91.0$

في دراسة قام بها Ibrahim S. Al-Mutaz و و Mohammad A. Al-Ghunaimi وحدات التتاضح العكسي بدرجات الحرارة العالية لتحلية

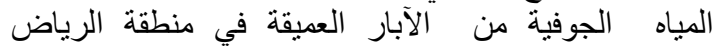

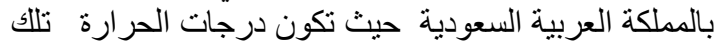

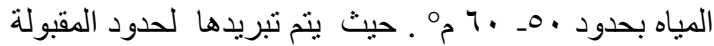

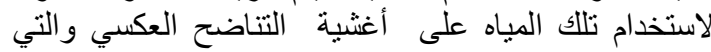

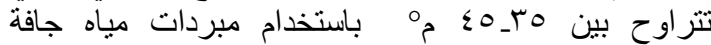
cascade chillers

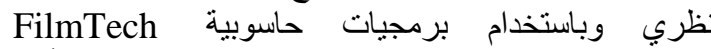
software بان ماء التخذية الاسخن يؤدي إلى نتائج وأداء

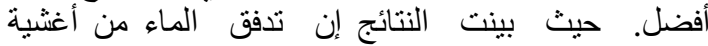

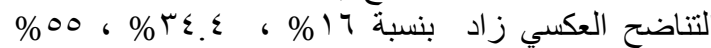

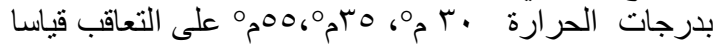

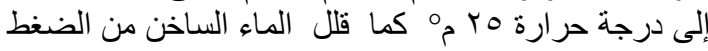

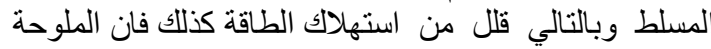
في الماء المنتج قد قلت في دراسة قام بها Mohamed Belkacem و آخرون

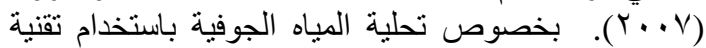

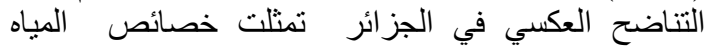

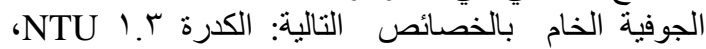

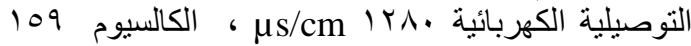

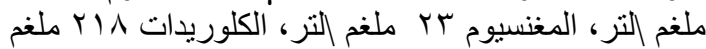

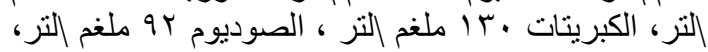

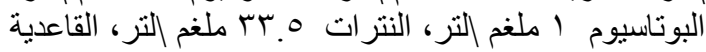

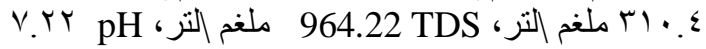

\begin{tabular}{|c|c|c|}
\hline المياه المحلاة & المياه الخام ( قبل التحلية) & الخاصية \\
\hline$r V \cdot$ & $0 \leqslant \ldots$ & التوصيلية الكهربائية MS/cm \\
\hline 6.3 & 6.8 & pH \\
\hline $1 \varepsilon$. & rqT. & 1 TDS \\
\hline ro & $V \varepsilon$ & العسرة الكلية بدلالة CaCo3 \\
\hline$\varepsilon$ & IYA & الكالسيوم ملفم| \\
\hline r. & $1 \cdots . \wedge$ & المغنسيوم ملغم| \\
\hline$\varepsilon \wedge . \Gamma$ & A^r.Y & الصوديوم ملغم| \\
\hline 11.90 & $\mathrm{VAl}$ & الكلوريد ملغم| \\
\hline or.A & $1 \cdot 1 \cdot$ & الكبريتات ملفم| \\
\hline $1.1 \mathrm{~V}$ & Yo.ro & البوتاسيوم ملغم| \\
\hline A.r. & $4 \pi . \wedge$ & 1 \\
\hline$\cdot$ & $\because \cdot 1$ & 1 \\
\hline$\because \cdot r$ & $\because+r$ & الحديد ملغم| \\
\hline$\because \cdot Y V$ & $\because \cdot 7 \varepsilon$ & المنغنيز ملغم| \\
\hline
\end{tabular}

تم إجراء فحص دوري لعدة خصائص للمياه الجوفية

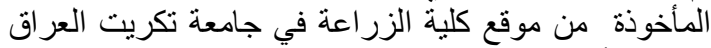

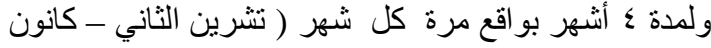

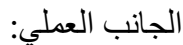

Characteristics of خصائص المباه الجوفية الخام :

Raw Groundwater 
Wastewater 20 $20^{\text {th }}$ Edition 1998 خصائص المياه الجوفية الخام وتواريخ اخذ النماذج
الأول ـ كانون الثاني- شباط) من بئر ييلغ عمق الماء

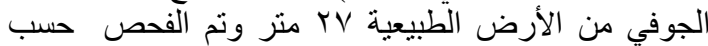
Standard الطرق القياسية لفحص المياه ومياه الفضلات Methods for the examination of Water and

\begin{tabular}{|c|c|c|c|c|c|}
\hline \multirow[t]{2}{*}{ طريقة الفحص } & تاريخ العينة & تاريخ العينة & تاريخ العينة & تاريخ العينة & \multirow{2}{*}{ الخاصية والوحدات } \\
\hline & 11 & 11 & 11 & 11 & \\
\hline $\begin{array}{c}\mathrm{pH}-\text { meter } \\
\text { digital }\end{array}$ & 7. $V$ & V.I & V.7 & V.V & $\mathrm{pH}$ \\
\hline $\begin{array}{c}\text { Temp.-meter } \\
\text { digital }\end{array}$ & Tr & TH.Y & Tr. & Tr.T & درجة الحرارة مه \\
\hline $\begin{array}{c}\text { Turbidity meter } \\
\text { HANNA }\end{array}$ & $\cdot . \wedge$ & . & $\because \leqslant 1$ &. ro & الكدرة NTU \\
\hline TDS-meter & $17 \mathrm{~V}$ & $10 \ldots$ & $10 \leqslant$. & $1 \leqslant 0$. & TDS ملغمالتز TDS \\
\hline Ec- meter & To.. & YIE. & TOY. & $r \cdot \varepsilon \cdot$ & $\mu \mathrm{s} / \mathrm{cm} \mathrm{Ec}$ \\
\hline التسحيح باستخدام & $I V \cdots$ & IV.. & $1 \wedge \ldots$ & $17 \ldots$ & العسرة الكلية على شكل مaCo3 \\
\hline التسحيح باستخدام & $\varepsilon 0$. & $\varepsilon \wedge \cdot .9$ & 071 & $\varepsilon \wedge$. & الكالسيوم ملغمالتز \\
\hline الطريقة الحسابية & $1 \varepsilon$. & $M 1.0$ & 97.7 & $9 V . Y$ & المغنسيوم ملغمالتر \\
\hline $\begin{array}{c}\text { التسحيح مع حامض الكبريتيكة } 0.02 \mathrm{~N} \\
\text { البرتقالية المثيلة }\end{array}$ & 17. & 17. & $r \ldots$ & $r \ldots$ & القاعدية على شكل ملنمل CaCo3 \\
\hline نترات الفضحة باستخدام التشرومات & rᄉ. & $r \leqslant q . \varepsilon$ & rq9.A & r99.1 & الكلوريدات ملغمالتر \\
\hline $\begin{array}{c}\text { Sulphate meter } \\
\text { Digital HANNA HI } \\
93751 \\
\end{array}$ & $r \ldots$ & $0 \ldots$ & $\varepsilon .0$ & r70 & الكبريتات ملغم التر \\
\hline Flame photometer & 70.710 & NI. Yo & VY.YO & $7 . \wedge V$ & الصوديوم ملغمالتر \\
\hline Atomic absorption & $\cdot$ & $\cdot$ & $\cdot$ & $\cdot$ & الرصاص ملغمالتز \\
\hline Atomic absorption &.$r$ & $\cdot r$ & $\cdot r$ & $\cdot \wedge$ & الحديد ملغمالتر \\
\hline
\end{tabular}

Reverse Osmosis ROSA 72 الأمريكية وهو System Analysis -:

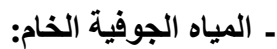

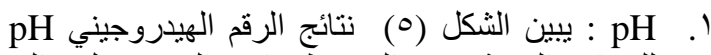

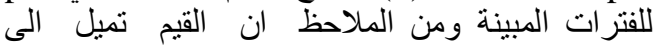

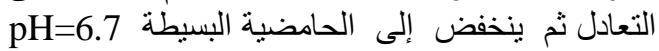

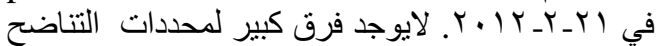

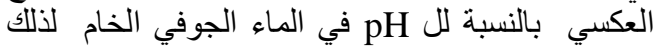

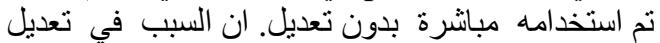

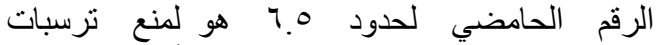
كاربونات الكالسيوم وتحسين رفض الأملاح (وضاح

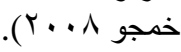

ثانيا: الجهاز المستخدم

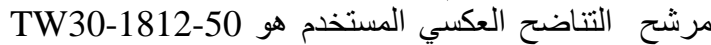
و المبين في الثكل رقم (乏 ).

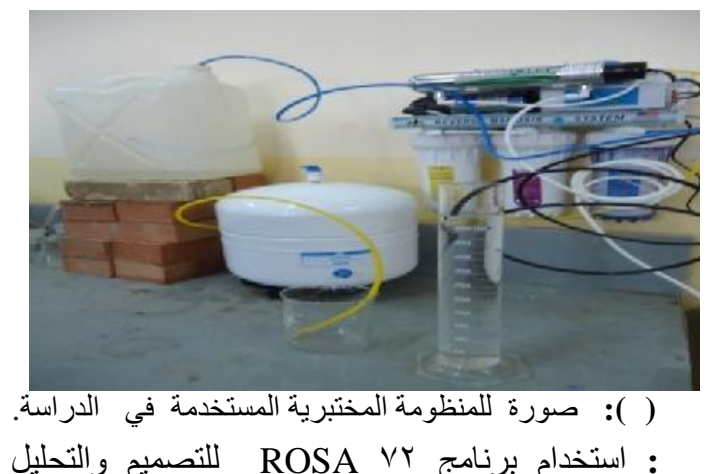

لمنظومات التناضح العكسي وهو من إصدار شركة ROW 


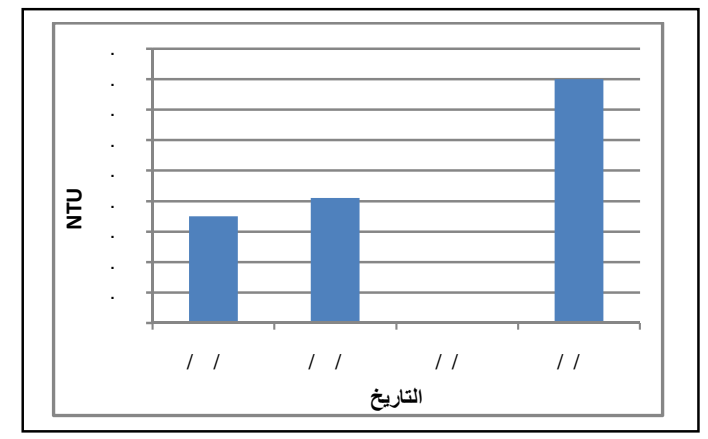

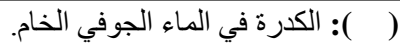

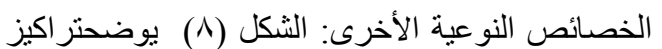

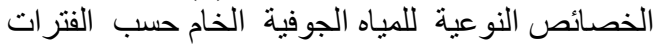

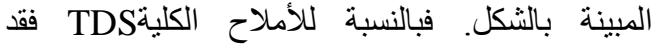

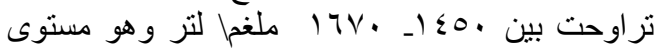

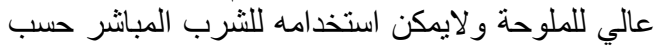

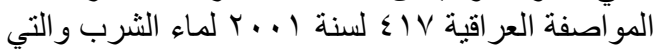

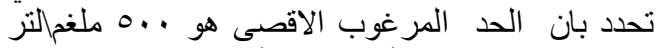

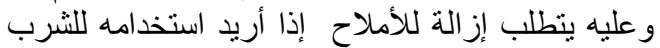

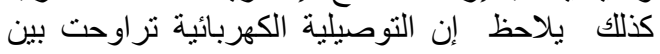

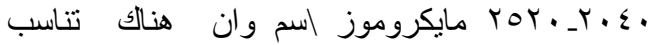
خطي بين الأملاح الذائبة الكلية TDS والتيكان التوصيلية

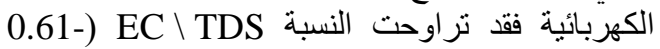

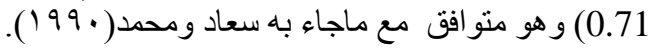
يلاحظ مستويات عالية للعسرة الكلبة في الماء الجوفي لـادي

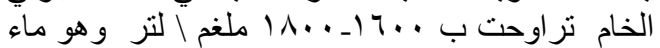

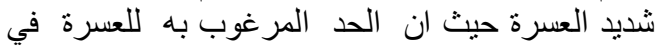

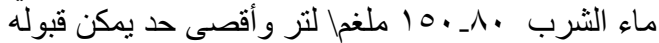

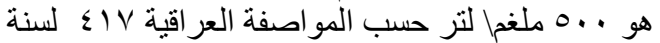

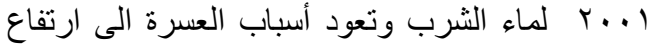

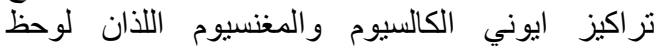

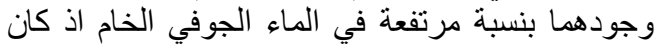

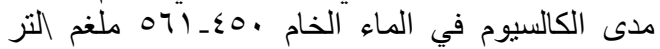

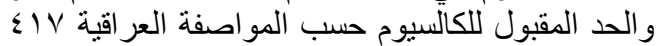

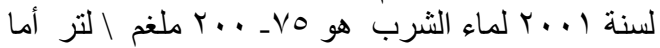

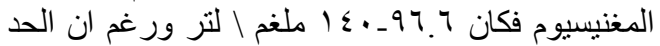

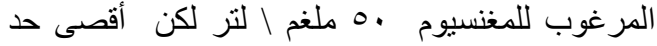

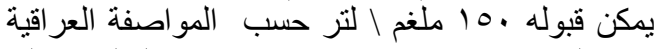

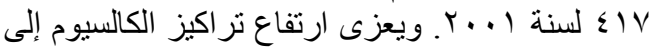

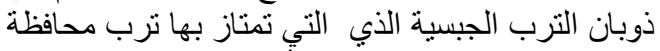

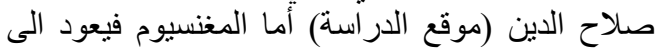

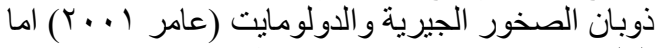

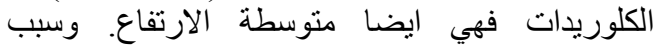

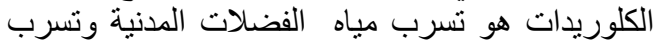

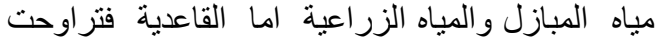

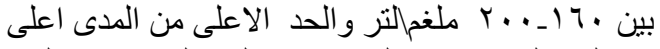

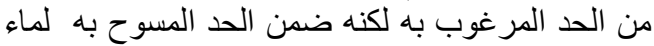

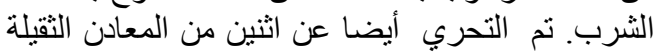

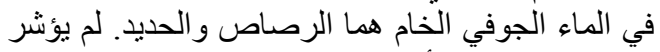

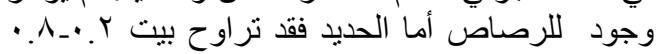

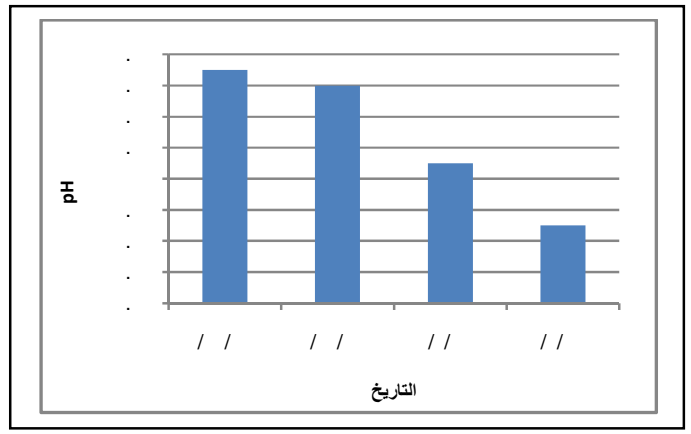

( ) ( ) الرقم الهيدروجيني للماء الجوفي الخام.

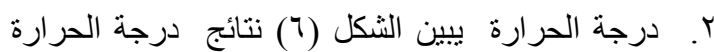

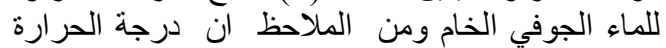

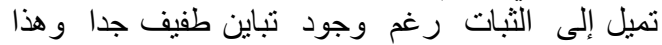

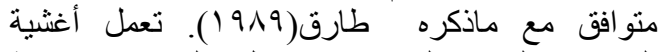

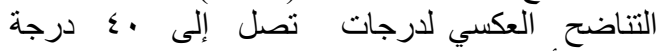
مئوية وأكثر لكن كفاءة الإزالة تقل مع ارتفاع دات درجة الحر ارة (Ibrahim and Mohammad 2001).

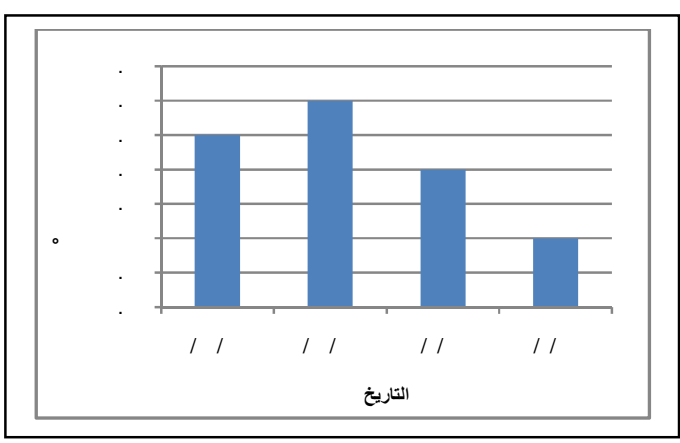

( ) ( ) درجة الحرارة للماء الجوفي الخام.

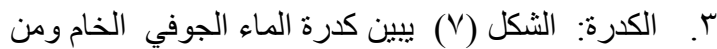

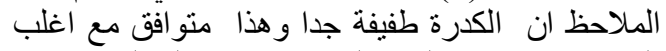
الدراسات. من ان المياه الجوفية تنميز بقلة الكدرة كون الكئح

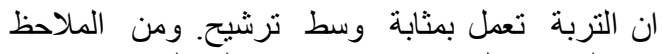

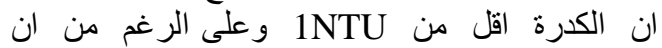

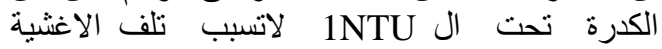

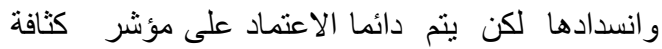

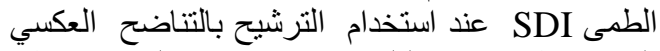

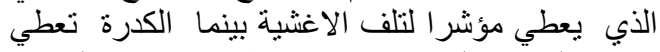

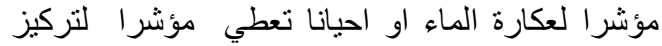

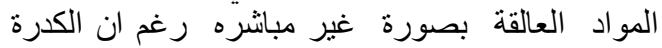

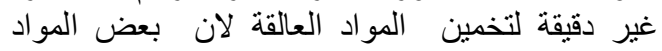

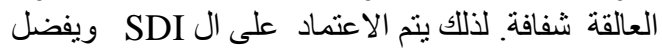

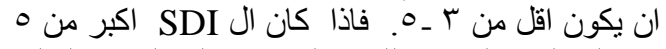

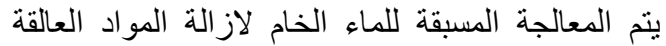

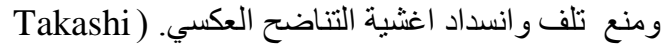

.(Asano et.al, 2007 


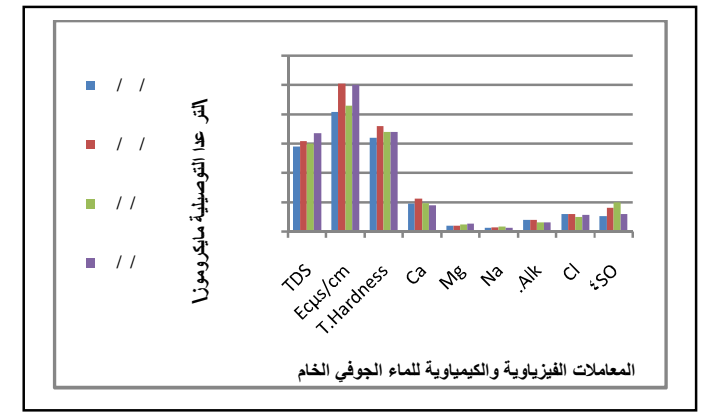

( ) ( ) المعاملات الفيزياوية والكيمياوية للماء الجوفي الخام.

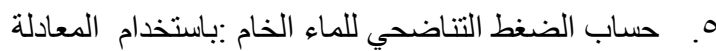
رقم (1) يكون الضغط التناضحي كما مبين في جدول الفياه

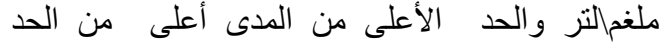

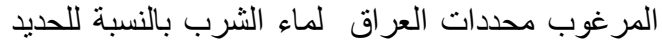

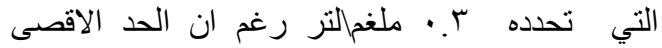

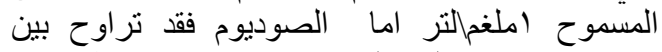

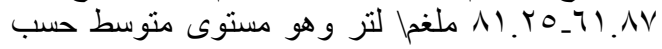

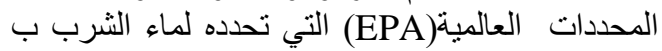

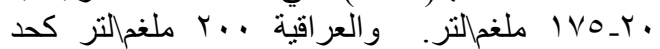

اقصى. (ق. (ق)

\begin{tabular}{|c|c|c|c|c|}
\hline$($ bar) $\pi$ & (Psi) $\pi$ & $\begin{array}{ll} & \text { TDS }\end{array}$ & ○ & التاريخ \\
\hline 1.10 & 17.07 & $1 \leqslant 0$. & rr.r & $r .11 / l 1 / r$. \\
\hline I.Y & IV.T. & $10 \leqslant$. & rr. & $r .11 / K / Y T$ \\
\hline 1.11 & $1 V .1 T$ & $10 \%$ & $r r . r$ & $r . I T / T / T r$ \\
\hline I.r & 19.7 & $17 \%$. & $r \mu$. & $Y \cdot \mid r / Y / Y)$ \\
\hline
\end{tabular}

ال TDS تطلب التغلب علية بضغط إضافي ليتم إزالة

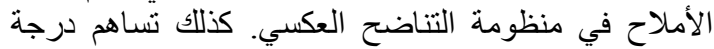
الحرارة في زيادة الضغط التخاضح التخحي وبالتالي تكون كفاءة الإزالة لمنظومة التناضح العكسي اقل في إزالة الألمالاح في

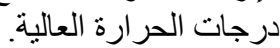
ثانياً: تثغيل المنظومة العالة ومواصفات (Retentate) Brine ( ) permeate :

تم تثغيل منظومة التتاضح العكسي لتحلية وإز الة الأملاح في المياه الجوفية ولمعرفة الخصائص الكمية والنوعية للماء النياء الدعالج premeate والمرفوض brine وكما مبين بالجدول

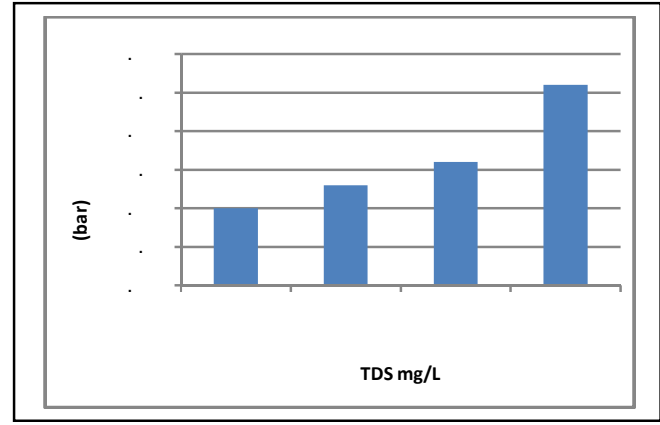

( ) ) : الضغط التناضحي نسبة إلى الأملاح الذائبة الكلية.

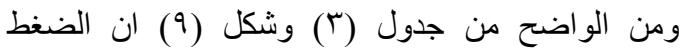
التناضحي يتناسب طرديا مع ال TDS و هذا يعني كلما زاد

\begin{tabular}{|c|c|c|c|}
\hline معدل تصريف الماء & معدل تصريف \قيقة & معدل تصريف الماء الداخلة & التاريخ \\
\hline .479 & $\because$ IT & ·. & $r \cdot \| / / r / r T$ \\
\hline.$r V T$ & $\because Y V$ & . $\varepsilon$ & $T \cdot|r / T / K|$ \\
\hline
\end{tabular}

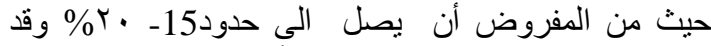

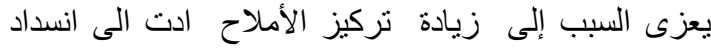
جزئي في المسامات وبالتالي قلل من الماء الماء المعالج المتدفق

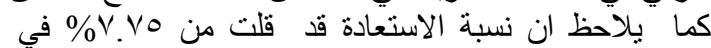

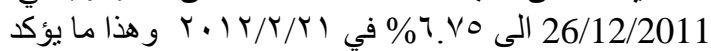

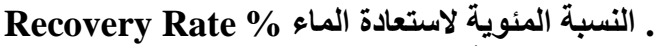
وعلى هذا الأساس يكون معدل استعادة الماء النعاء (النسبة

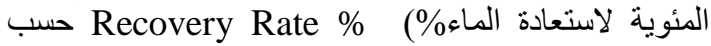

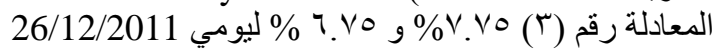
و21/2/2012 على التعاقب. ويلاحظ إن نسبة الاستعادة قليل 


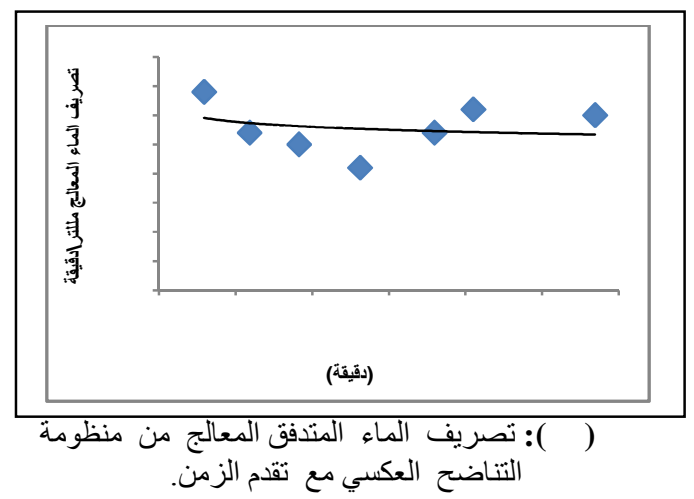

السبب السابق. بمكن زيادة المتدفق بزيادة الضغط المسلط بشرط أن يتحمل الغشاء إي زيادة في الضغط بلئ المسلط.

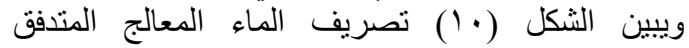
permeate

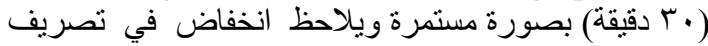

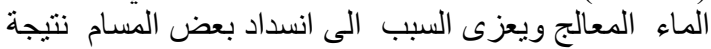
التصاق الايونات في سطح الغشاء.

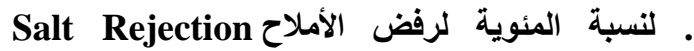

Percentage of

بالاستناد إلى المعادلة رقم (ץ) فان الجدول ( 5) يبين

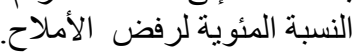

\begin{tabular}{|c|c|c|c|c|}
\hline كمية الأملاح المقيزالة & النسبة المئوية لرفض & تركيز TDS & تركيز TODS ماء التذذية & التاريخ \\
\hline 041 & QY.Y. & IT. & $10 \leqslant$. & $T \cdot 11 / T / Y T$ \\
\hline 097 & N9.YY & $1 \wedge$. & $17 \%$. & $r \cdot \mid r / T / Y)$ \\
\hline
\end{tabular}

ان ارتفاع تراكيز الأملاح الكلية والعسرة في الماء

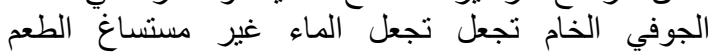

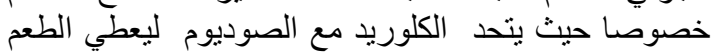

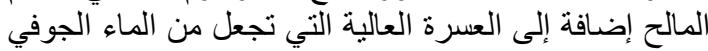

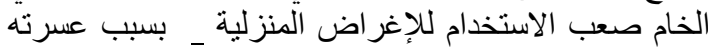

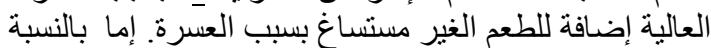

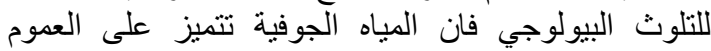

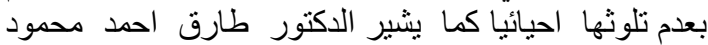

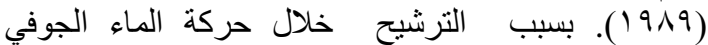
بالتربة الذي يزيل اغلب البكتريا و الإحياء المجهرية البهاء

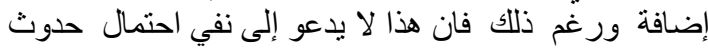

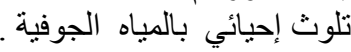

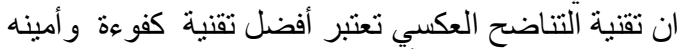
وموثوقة في إزالة الأملاح و المواد العالقة والبكتريا

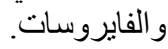
تم إجر اء تحليل نوعي للمياه الجوفية المرشحة من خلال منظومة التناضح العكسي (Permeate) وكانت النتائج كمال مبين في الجدولين 7 و V.
ومن الملاحظ إن هناللك انخفاض طفيف في النسبة المئوية

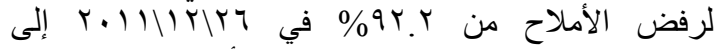

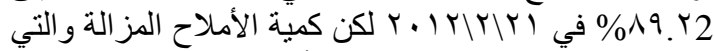

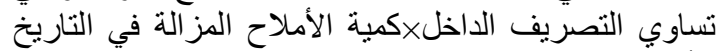
الأخير هي اكبر كما مبين بالجدول (ه) (ه).

\section{الفيزياوية والكيميائية للماء الجوفي}

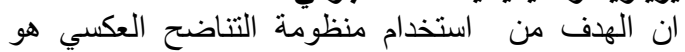

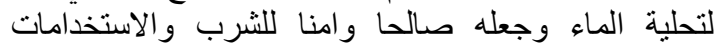

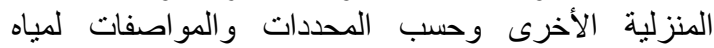

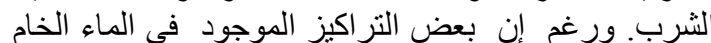
تقع ضمن الحدود المقبولة لماء الثرب الثرب إلا إن البعض الكض الأخر

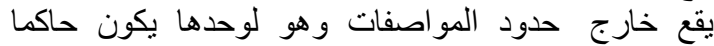

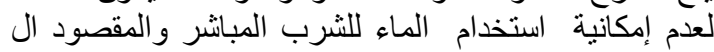
و والعسرة و الكالسيوم إما الكلوريدات و والكبرينات

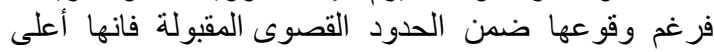

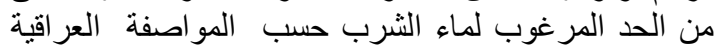

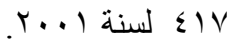




\begin{tabular}{|c|c|c|c|c|}
\hline كفاءة الازالة \% & $\begin{array}{l}\text { الماء النقي المرشح } \\
\text { Permeate }\end{array}$ & الماء الجوفي الخام & الوحدة & الخو اص \\
\hline $9 Y .4$ & Ir. & $10 \leqslant$. & ملغمالتر & TDS \\
\hline $91 . Y 7$ & rr. & ror. & $\mu \mathrm{s} / \mathrm{cm}$ & التوصيلية الكهربائية \\
\hline or. 70 & .19 &.$\leqslant 1$ & NTU & الكدرة \\
\hline 90.00 & $\Lambda$. & $11 \ldots$ & ملغمالتر & 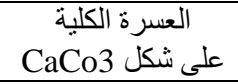 \\
\hline $9 V_{.} .$. & 7 & r.. & ملغمالتر & القاعدية \\
\hline $97 . \leqslant T$ & $r$. & 071 & ملغمالتر & الكالسيوم \\
\hline 91.90 & V.VV & 97.7 & ملغمالتر & المغنيسيوم \\
\hline qE.YV & rY.q & rq9.A & ملغمالتر & الكلوريدات \\
\hline q.r. rV & rq & $\varepsilon .0$ & ملغمالتر & الكبريتات \\
\hline 97.47 & T.7YO & VY.YO & ملغمالتر & الصوديوم \\
\hline $1 \ldots$ & Nil & $\cdot r$ & ملغمالتر & الحديد \\
\hline
\end{tabular}

\begin{tabular}{|c|c|c|c|c|}
\hline كفاءة الازالة \% & $\begin{array}{l}\text { الماء النقي المرشح } \\
\text { Permeate }\end{array}$ & الماء لجوفي الخام & الوحدة & الخو اص \\
\hline AQ.YY & 11. & $17 \%$. & ملغمالتر & TDS \\
\hline$\wedge \wedge$. & r... & ro.. & $\mu \mathrm{s} / \mathrm{cm}$ & التوصيلية الكهربائية \\
\hline NI.YO & .10 & $\cdot . \wedge$ & NTU & الكدرة \\
\hline $9 \leq .11$ & $1 \ldots$ & IV.. & ملغمالتر & 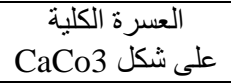 \\
\hline 97.10 & 7 & 17. & ملغمالتر & القاعدية الكلية \\
\hline $9 Y . \wedge 1$ & ru & $\leqslant 0$. & ملغمالتر & الكالسيوم \\
\hline $97.0 Y$ & $\varepsilon . \wedge 7$ & $1 \leqslant$. & ملغمالتر & المغنسيوم \\
\hline 94.71 & $T \varepsilon$ & rA. & ملغمالثر & الكلوريدات \\
\hline 10.77 & $\sum r$ & $r \ldots$ & ملغمالتر & الكبريتات \\
\hline VO.VY & 10.94 & 70.710 & ملغمالتر & الصوديوم \\
\hline $1 \ldots$ & Nil & $\cdot r$ & ملغمالتر & الحديد \\
\hline
\end{tabular}

TDS الكلية

الجدول (^) يبين تركيز الTDST في الماء المرفوض مقارنة

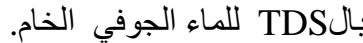

\begin{tabular}{|c|c|c|}
\hline المرفوض الجوفي ملغمالتر & للماء الجوفي الخام ملغرالتر & التاريخ \\
\hline 104. & $10 \leqslant$. & $r \cdot \| I I Y I r q$ \\
\hline $17 \ldots$ & $17 \%$. & $T^{\prime} \cdot|Y| Y|Y|$ \\
\hline
\end{tabular}

التناضح العكسي.

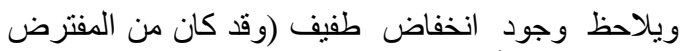

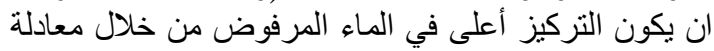

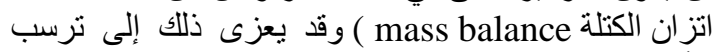

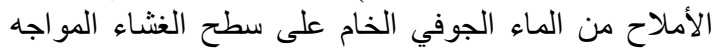

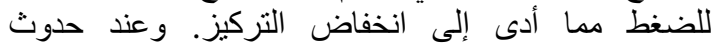

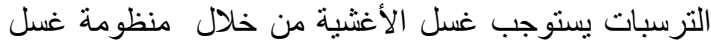

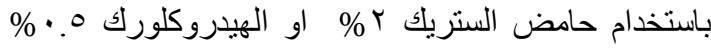

ومن خلال النتائج المستحصلة يلاحظ ارتفاع كفاءات الإتهاء

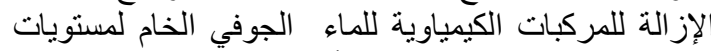

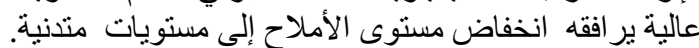

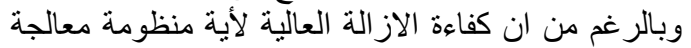

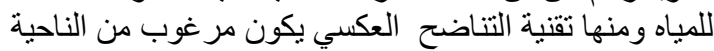

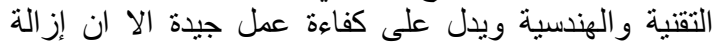

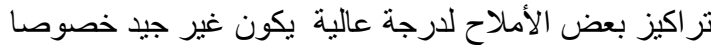

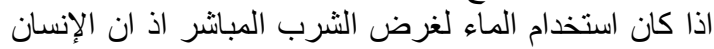

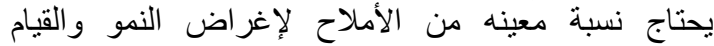

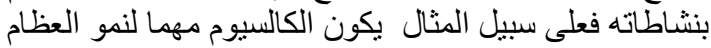

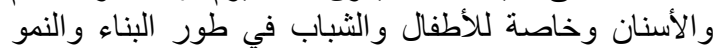

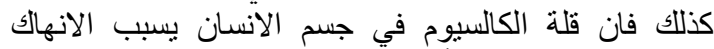

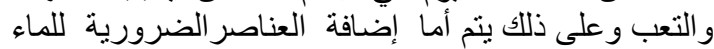

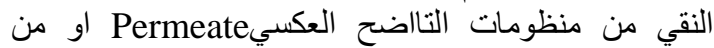
الناحية العملية يتم إجر اء مزج جاء جزء من الماء الماء الخام بمعادلة

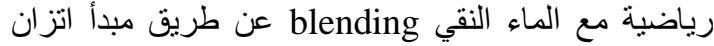

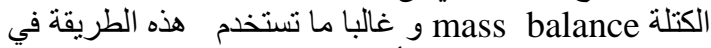
مصانع إنتاج المياه المعبأة bottled water لكونها علية علية

و اقتصادية (C. C. Lee, and Shun Dar Lin Y..V ) 


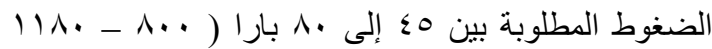

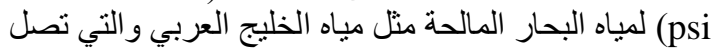

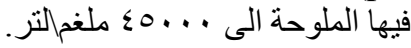

• تنفيذ برنامج 72 ROSA لتصميم منظومة التناضح

تم تتفيذ برنامج 72 ROSA بادخال البيانات المطلوبة حسب الخصائص التي تم الحصول عليها عمليا وكانت النتائج

حسب التقرير النهائي للبرنامج وكما مبين يالجدول (

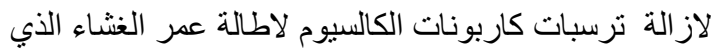

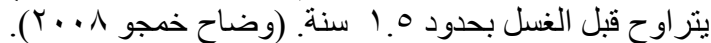

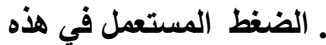

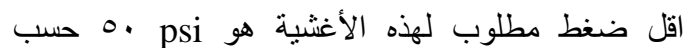

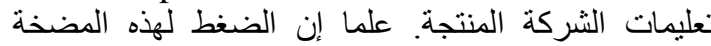

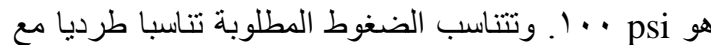

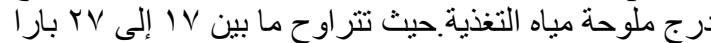

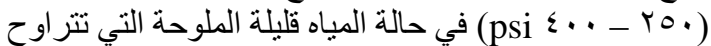

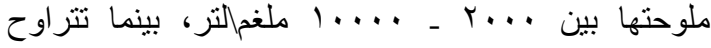

Project Information:

Case-specific:

System Details

Feed Flum to Stage 1

Raw Water Huw tos System

Feed hresure

Flow Factor

Chem. Dsse (1006 H2504)

Total.Actite.Area

Wazter Classificatina: Well Water SOD $<3$

\begin{tabular}{|c|c|}
\hline $0.30 \mathrm{~m}^{3} / \mathrm{h}$ & Pass 1 Permeate Fion \\
\hline $0.30 \mathrm{~m}^{3 / 2}$ & Pass 1 Recovery \\
\hline $7.30 \mathrm{bar}$ & Feed Temperature \\
\hline 0.85 & Feed TDS \\
\hline $0.00 \mathrm{mg}]$ & Number of Elements \\
\hline $260 \mathrm{y}^{2}$ & Arernge Pass 1 Fur \\
\hline
\end{tabular}

Stage Elemert FPV $\neq$ Ele

Feed flow
$\left(\mathrm{m}^{\mathrm{I}} / \mathrm{h}\right)$
Feed

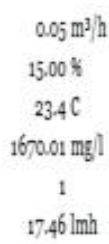

$17.46 \mathrm{lmh}$ osmotic Pressure:

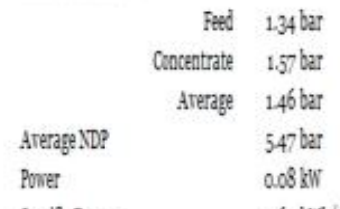

Specific Energy $\quad 1.69 \mathrm{kWh} / \mathrm{m}^{3}$

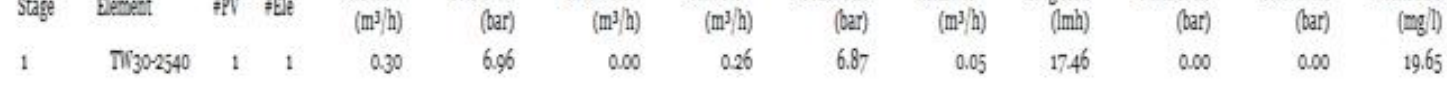

( ) ( ) (التقرير النهائي لبرنامج ROSA72.

المتدفق (المعالج) يقل مع تقدم الزمن خلال فترة

ترشيح و احدة. ثانياًـ التوصيات: ترنيات

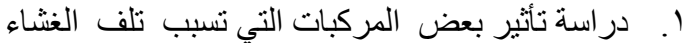

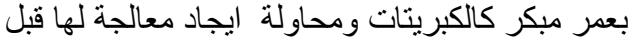
الترشيح.

r. براسة إنتاجية الماء المتدفق من منظومة التناضح

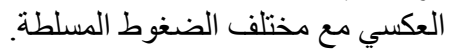
r. در اسة إمكانية الاستفادة من المياه الجوفية الخام لإنتاج

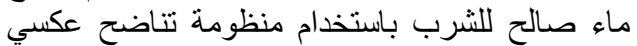

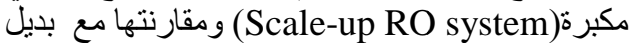
منمثل نصب وحدة إسالة في الجامعة لمعالجة المياه السطحية (Compact Unit) من ناحية الكلفة ونو عية الماء وكميته و الصيانة.

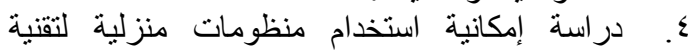

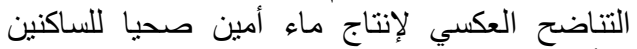
بالأرياف و الذين بعتمدون على الإنى الماء الجين الجوفي كمصدر

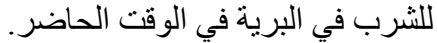
زيادة الوعي بتحلية الماء الجوفي الوفي باستخدام التناضح

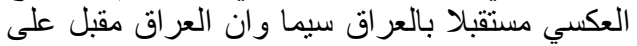

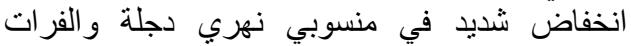

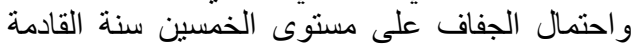
بسبب إنثاء السدود في تركيا مع زيادة النمو السكاني
الاستتتاجات و التوصيات

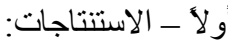
ا الـ ارتفاع تر اكيز المياه الجوفية الخام في موقع جامعة

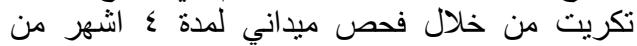

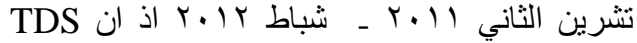

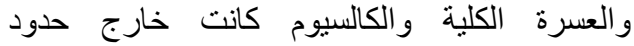
المواصفة العراقية لماء الشرب. الثراء لايمكن استخدامه للشرب المباشر وينطلب القيام بالتحلية.

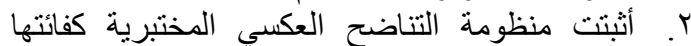
العالية _ بتقليل المكونات الفيزياوية و الكيمياوية ليكون المئية

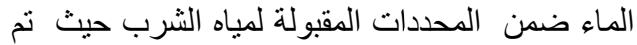

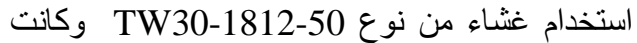
نتائج كفاءة الازالة كالاتي: للأملاح الذائبة الكلية TDS

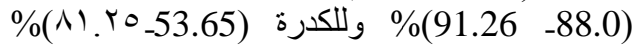

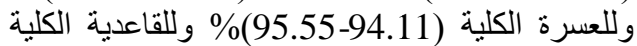
\%

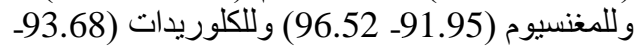

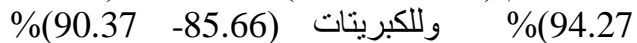

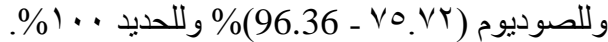

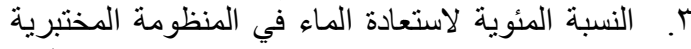

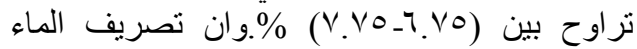


Treatment by reverse Osmosis Desalination Journal 206 100-106.

Sawyer and McCarty (1978). "Chemistry for Environmental McGraw-Hill Company page 62 Engineering" $3^{\text {rd }}$ Edition.

Standards Methods for the examination of water and wastewater (1998). $20^{\text {th }}$ edition.

Takashi Asano, Franklin L. Burton, Harold L. Leveren, Ryujiro Tsuchihashi,and George Tchobanoglous (2007). "Water Reuse" First Edition McGrawHill Company

طارق احمد محمود ( 1919). علم وتكنولوجيا البيئة - كلية

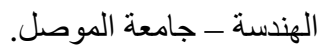

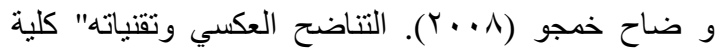

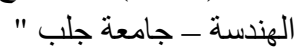

محمد المعالج وصالح بوقثة ـو اقع و آفاق تحلية المياه في

الوطن العربي ومدى إمكانية استخدام الطاقات

المتجددة - جامعة الدول العربية -إدارة برنامج العامج

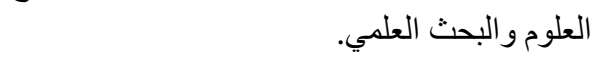

سعاد عبد عباوي ومحمد سليمان حسن (.99 199 ) - الهندسة

العملية للبيئة -فحوصات الماء - جامعة الموصل.

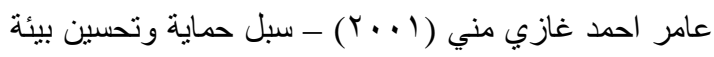
المصانع -الطبعة الثالثة.
في العراق الأمر الذي يؤدي الى زيادة الطلب على

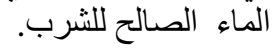

Abbas Hadi Abbas (2016). "RO softens brackish groundwater in Tikrit, Iraq". Water Reuse \&Desalination. Vol. (7) Issue (1).

C.C. Lee and Shun Dar Lin (2007). "Handbook of Environmental Engineering Calculations, 2nd edition McGraw-Hill Company.

G.R. Lashkaripour and M. Zivdar (2005). " Desalination of brackish ground water in Zahdan City in Iran " Desalination Journal 177 .1-5.

Ibrahim, S. Al-Mutaz and Mohammad A. AlGhunaimi (2001). Performance of Reverse Osmosis Units at High Temperatures The IDA World Cogress on Desalination and Water Reuse, Bahrain,

Maria Dina Afonsoa, Jamal 0. Jaberb, Mousa, and S. Mohsenb (2004). "Brackish groundwater treatment by reverse osmosis in Jordan" Desalination Journal $164 \quad 157-171$ www.eisevteccomllocam/desal

Mohamed Belkacem, Sadia Bekhti and Kenza Bensadok (2007). "Groundwater

\title{
Softening of Groundwater in Tikrit University by Using Reverse Osmosis Membrane Technique
}

\author{
Abbas Hadi Abbas \\ Dept. of Civil Engineering - College of Engineering - University of Samarra
}

\begin{abstract}
:
This research is accomplished to the soften of groundwater at Tikrit University site by using reverse osmosis (RO) membrane system. RO type TW $30-1812-50$ is used in this research. The system achieved water recovery rate percentage between 6.75\%-7.75 \% and salt rejection percentage ranged between $89.22 \%-92.2 \%$.Also the results showed high removal efficiency for physical and chemical concentrations of raw groundwater for TDS (89.22-92.2 \%, EC (88.*91.29)\%, Turbidity (53.65-81.25)\%. Total Hardness (94.11-95.55\%, Alkalinity (96.25-97. $\cdots) \%$, $\mathrm{Ca}(92.88-96.43) \%, \mathrm{Mg}(91.95-96.52) \%, \mathrm{Cl}(93.68-94.27) \%, \mathrm{SO} 4(85.66-90.37) \%, \mathrm{Na}(75.72-$ $96.36) \%$, and $\mathrm{Fe} 100 \%$. ROSA 72 software program is used in designing of reverse osmosis then the results were satisfied and accepted.
\end{abstract}

